\section{Des cadhérines à la quantification de l'adhérence intercellulaire}

\author{
Sylvie Dufour, Yeh-Shiu Chu, Jean-Paul Thiery
}

> L'adhérence est une propriété physique qui avait impressionné Aristote 350 avant JC. Son observation a conduit récemment à déterminer le mécanisme d'adhérence absolument remarquable du coussin plantaire du gecko [1]. L'adhérence cellulaire est un processus fondamental chez tous les organismes. Dès la fin du XIX siècle, les embryologistes se sont intéressés aux mécanismes de reconnaissance et d'adhérence intercellulaire. Plusieurs approches fondées sur des méthodes de dissociation des tissus par la trypsine ont permis de mettre en évidence deux mécanismes d'adhérence distincts entre cellules. Le premier, ne dépendant pas du calcium, a conduit à la découverte de la première molécule d'adhérence intercellulaire $\mathrm{N}$ CAM, prototype des molécules d'adhérence de la superfamille des immunoglobulines [2]. Le deuxième mécanisme, dépendant du calcium, est contrôlé par une autre classe de récepteurs membranaires appelés cadhérines classiques [3]. La $\varepsilon$-cadhérine a été l'une des premières cadhérines identifiées. Elle est exprimée dès les premiers stades du développement et son expression devient importante dans tous les épithéliums. Aujourd'hui, le répertoire des cadhérines s'est considérablement complexifié [4]. On distingue les cadhérines classiques de type I qui comme la $\varepsilon$-cadhérine maintiennent une forte cohésion des cellules au sein des tissus et peuvent aussi intervenir dans le remodelage tissulaire ou de migration de cohortes cellulaires. Les cadhérines classiques de type II, bien que ressemblant à celles de type I, sont au contraire très souvent exprimées dans des tissus peu cohésifs ou de type mésenchymateux et aussi par des cellules isolées en migration.

Les cadhérines classiques sont composées d'une partie extracellulaire constituée de cinq domaines de type immunoglobuline, capable d'interagir avec une molécule identique portée à la surface de la cellule voisine. Elles possèdent aussi un domaine transmembranaire et une région cytoplasmique capable de recruter des connecteurs au cytosquelette d'actine comme la $\beta$ - et l' $\alpha$-caténine, et d'activer des cascades de signalisation cellulaire. Les cadhérines sont bien plus que de simples « colles » cellulaires car l'activation du signal d'adhérence dépendant des cadhérines contrôle la polarité et la forme des cellules, module l'expression génique et régule la prolifération, la survie et la différenciation cellulaire [5]. Plusieurs travaux suggèrent que les cadhérines de type II seraient moins adhérentes que les cadhérines de type I et ainsi leur expression plus compatible avec les remodelages et migrations cellulaires [6]. II nous est donc apparu essentiel de quantifier par des méthodes physiques le mécanisme d'adhérence intercellulaire. L'objectif à court terme étant de mieux comprendre de quelle manière un processus d'adhérence se développe; quelle est la contribution des différents connecteurs et en particulier en quoi les différentes cadhérines confèrent des interactions stables ou labiles. La compréhension de ce processus est essentielle dans l'étude de nombreux états physiopathologiques.
Nous avons utilisé une technique dérivée d'une méthode développée par E. Evans [7], et manipulé des cellules individuelles en suspension par des micropipettes pour former des doublets adhérents et déterminer la force nécessaire pour les séparer (Figure IA). Cette technique a l'avantage de permettre un contrôle parfait du temps de contact avant de procéder à la séparation du doublet cellulaire. Elle permet ainsi d'analyser la cinétique de développement de l'interaction en fonction du temps de contact et de déterminer l'influence de nombreux paramètres sur ce processus. Cette technique ne mesure pas la force d'interaction à l'échelle moléculaire entre récepteurs présents à la surface cellulaire ni la force d'adhérence intercellulaire, toutes deux étant des concepts physiques erronés. Une force ne peut d'ailleurs être mesurée directement sur des protéines en interaction mais seulement un paysage énergétique. La technique des micropipettes permet de mesurer la force nécessaire pour séparer des cellules en contact, dite force de séparation, et utilisée comme un indicateur quantitatif de l'adhérence intercellulaire.

Lorsque des cellules qui expriment uniquement comme récepteurs d'adhérence des $\varepsilon$-cadhérines sont mises en contact par les micropipettes, elles adhèrent très rapidement. Après quelques secondes, il est possible de mesurer une force de séparation de quelques nanoNewtons $(\mathrm{nN})$, qui augmente rapidement et linéairement avec le temps puis plus lentement jusqu'à obtention d'un plateau après une heure de contact où la force de séparation, qui atteint $220 \mathrm{nN}$, n'évolue alors que très peu en fonction du temps (Figure 1B). L'intensité de la force de 


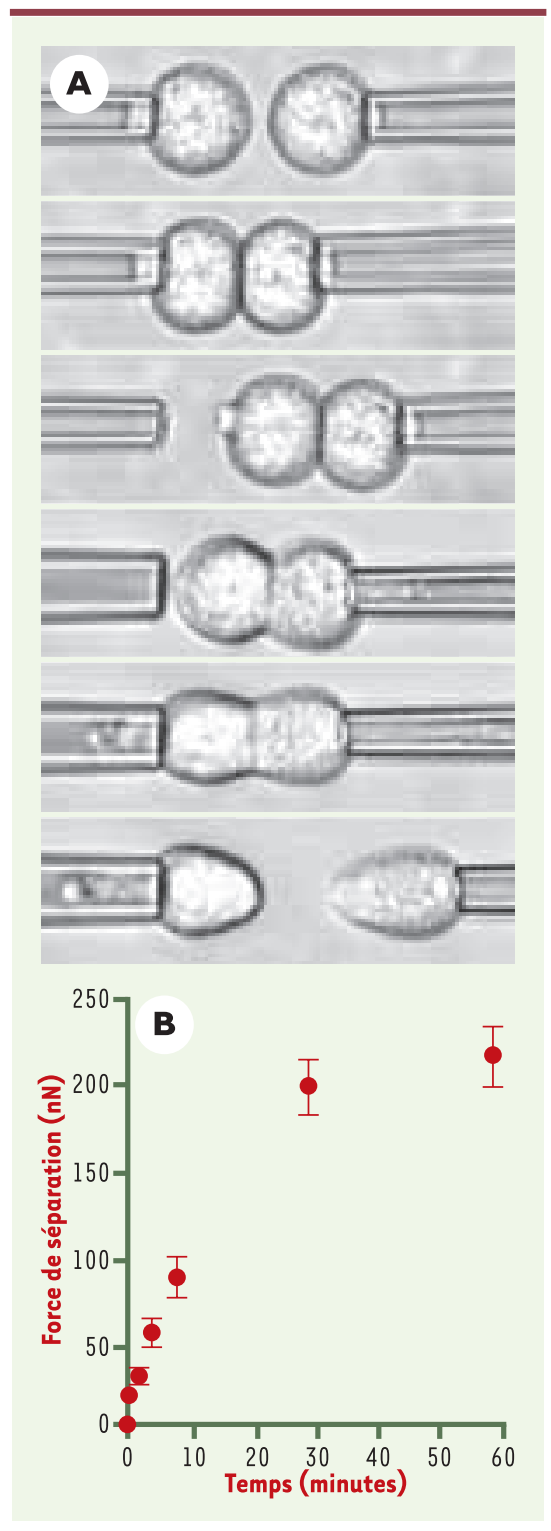

séparation à un temps donné de contact varie en fonction de l'expression de la $\varepsilon$ cadhérine à la surface. Ces résultats indiquent clairement que la présence et le niveau d'expression de $\varepsilon$-cadhérine à la surface sont les paramètres clés contrôlant le déclenchement et le développement de l'adhérence intercellulaire [8]. Au cours du temps, le nombre de $\varepsilon$ cadhérine augmente au niveau du contact intercellulaire de même que la $\beta$-caténine et l'actine (Figures $2 A$ et $B$ ). Nous montrons que la réorganisation $d u$ cytosquelette d'actine est requise pour le renforcement de l'interaction inter-
Figure 1. Méthode de mesure de la force de séparation. A. De haut en bas et de droite à gauche: les cellules exprimant la $\varepsilon$-cadhérine sont mises en contact et forment un doublet adhésif ensuite maintenu sous forte aspiration par la pipette de droite. Des étapes successives d'augmentation de l'aspiration dans la pipette de gauche et d'écartement des pipettes sont effectuées jusqu'à l'obtention de la séparation du doublet. Connaissant l'aspiration appliquée à gauche au moment de la séparation et le rayon interne de la pipette, il est possible de calculer la force de séparation. B. La force (exprimée en nanoNewton) nécessaire pour séparer des cellules est fonction de la durée du contact entre cellules (d'après [8]).

cellulaire. En effet, en présence de réactifs inhibant la polymérisation de l'actine ou bien en remplaçant des cadhérines sauvages par des cadhérines mutées, délétées des sites de liaison aux connecteurs cytoplasmiques de l'actine (Figure 2C), il y a déclenchement de l'adhérence entre cellules, mais celle-ci ne peut se renforcer au cours du temps; la force de séparation qui est mesurée stagne à quelques $\mathrm{nN}$ (Figure 2D). Les petites GTPases Rac et CDC 42 participent à la réorganisation de l'actine au niveau des contacts intercellulaires [9]. Nous montrons que ces protéines sont clairement impliquées dans le renforcement de l'interaction cellulaire dépendante des cadhérines puisque l'expression de formes mutantes de ces GTPases, capable d'exercer une activité dominante négative ou positive sur la fonction des protéines endogènes, abolit ce processus.

Les recherches menées par notre équipe ont aussi permis de montrer que certaines cadhérines de type II sont beaucoup moins adhérentes que celles de type I (données non publiées). Ces travaux vont permettre aussi de définir le rôle de chaque connecteur dans la mise en place des micro-domaines d'adhérence et en particulier d'aborder la question de la reconnaissance cellulaire. Enfin, des travaux sont engagés pour comprendre la

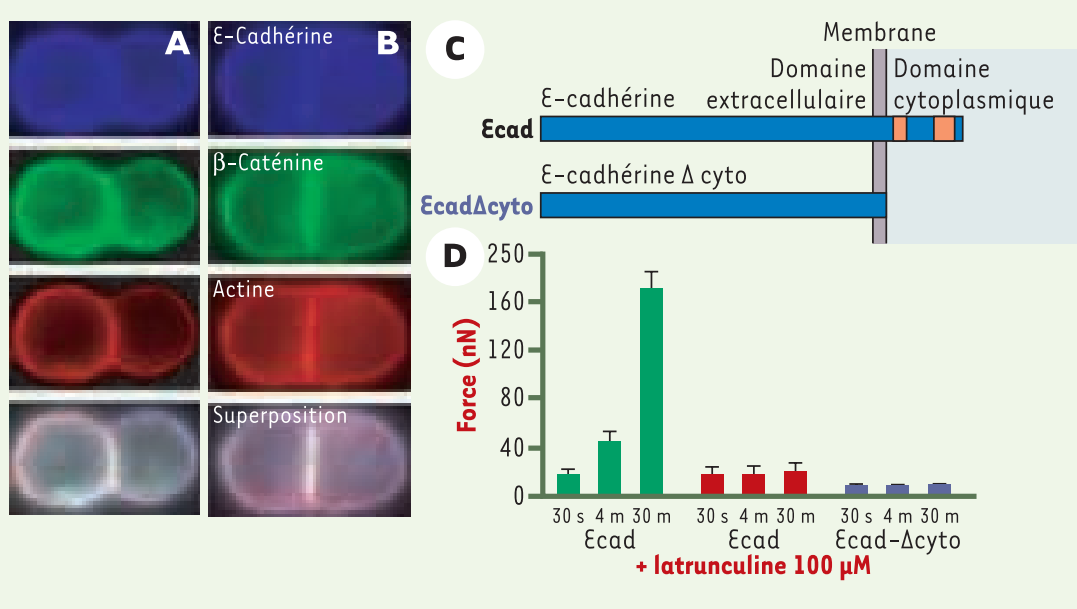

Figure 2. La connexion des cadhérines au cytosquelette d'actine contrôle le renforcement de l'interaction intercellulaire. $A-B$. La cadhérine (marquée en bleu) est localisée au contact intercellulaire du doublet et recrute la $\beta$-caténine (en vert) et l'actine (en rouge). Ces protéines sont enrichies au contact durant le développement de l'adhérence intercellulaire ( $A: 4$ minutes; $B$ : 30 minutes) (d'après [8]). C. Structure simplifiée de la $\varepsilon$-cadhérine sauvage ( $\varepsilon$ cad) et de la $\varepsilon$ cadhérine mutante ( $\varepsilon$ cad $\Delta$ cyto) ayant perdu son domaine cytoplasmique et les sites de liaison aux caténines (en orange). D. L'expression de $\varepsilon$ cad $\Delta$ cyto dans les cellules ne permet pas le renforcement de l'adhérence intercellulaire (barres bleues) comparé à ce qui est obtenu avec la cadhérine sauvage (barres vertes). L'incubation des cellules avec un bloquant de la polymérisation de l'actine (latrunculine A) produit le même effet (barres rouges). 
mise en place des structures d'adhérence des cellules épithéliales. Nos premiers résultats montrent le rôle coopérateur des nectines dans la formation des jonctions adhérentes [10]. $\diamond$

From cadherins to the quantification of cell adhesion

\section{RéFÉRENCES}

1. Autumn K, Sitti M, Liang YA, et al. Evidence for van der Waals adhesion in gecko setae. Proc Natl Acad Sci USA 2002; 99: 12252-6.
2. Thiery JP, Brackenbury R, Rutishauser U, Edelman GM Adhesion among neural cells of the chick embryo. II: purification and characterization of a cell adhesion molecule from neural retina. J Cell Biol 1977; 252: 6841-5.

3. Takeichi M. Functional correlation between cell adhesive properties and some cell surface proteins I Cell Biol 1977; 75: 464-74.

4. Nollet F, Kools P, van Roy F. Phylogenetic analysis of the cadherin superfamily allows identification of six major subfamilies besides several solitary members. J Mol Biol 2000; 299: 551-72.

5. Wheelock MJ, Johnson KR. Cadherin-mediated cellular signaling. Curr Opin Cell Biol 2003; 15: 509-14.

6. Dufour S, Beauvais-Jouneau A, Delouvée A, Thiery JP. Differential function of $\mathrm{N}$-cadherin and cadherin- 7 in the control of embryonic cell motility. J Cell Biol 1999; 146: 501-16.
7. Evans $\varepsilon$, Leung A. Adhesivity and rigidity of erythrocyte membrane in relation to wheat germ agglutinin binding. J Cell Biol 1984; 98: 1201-8.

8. Chu YS, Thomas WA, Eder 0 , et al. Force measurements in $\varepsilon$-cadherin-mediated cell doublets reveal rapid adhesion strengthened by actin cytoskeleton remodeling through Rac and $\mathrm{Cdc} 42$. J Cell Biol 2004; 167: 1183-94.

9. Yap AS, Kovacs EM. Direct cadherin-activated cell signaling: a view from the plasma membrane. J Cell Biol 2003; 160: 11-6.

10. Martinez-Rico C, Pincet F, Perez $\varepsilon$, et al. Separation force measurements reveal different types of modulation of $\varepsilon$-cadherin-based adhesion by nectin1 and -3. J Biol Chem 2004; 280 : 4753-60.
NOUVELle

Les effets profibrosants
des peptides vasoactifs
dans le rein et les vaisseaux
passent-ils
par la transactivation
du facteur de croissance
épidermique (EGF)?

Martin Flamant, Jean-Claude Dussaule, Raymond Ardaillou
> Le rôle des peptides vasoconstricteurs, tels que l'angiotensine II (Ang II) et l'endothéline ( $દ T)$, dans la progression de la fibrose rénale et vasculaire est maintenant largement admis, l'argument principal étant le ralentissement de cette progression et, même, son inversion lorsqu'on inhibe les effets de ces peptides par des antagonistes spécifiques de leurs récepteurs. Le mécanisme de ces effets profibrosants commence à être connu. De nombreuses études tendent à prouver l'implication des facteurs de croissance, principalement le facteur de croissance épidermique (EGF, epidermal growth factor). Le récepteur de l'EGF ( $\varepsilon G F-R$, epidermal growth factor receptor) est transactivé au tout début de la cascade d'événements induite par l'Ang II ou l'ET, ce qui veut dire que la séquence d'effets cellulaires connue comme étant celle produite par l'£GF se substitue en partie ou s'ajoute aux voies classiques d'action de ces deux peptides. Ce processus est donc totalement différent d'une éventuelle induction $d u$ gène codant pour l'EGF- $R$ en présence d'Ang II ou d'ET.

\section{La transactivation d'عGF-R}

Les arguments en faveur de la transactivation d'EGF-R reposent soit sur l'utilisation d'agents inhibant l'activation de ce récepteur, soit sur celle de souches de souris mutantes, soit enfin sur celle d'inhibiteurs de la transcription du gène. EGF$\mathrm{R}$ est un récepteur à activité tyrosine kinase dont l'activation suppose une étape préalable de dimérisation, puis de phosphorylation. Les principaux inhibiteurs agissent en bloquant cette phosphorylation. Les plus utilisés sont le PD153035, l'AG1478, tous deux de la famille des quinazolines, et le gefitinib (Iressa $^{\circledR}$, ZD1839). Ces inhibiteurs entrent
Inserm U.702, Hôpital Tenon,

4 rue de la Chine,

75020 Paris, France.

martin.flamant@sls.aphp.fr

en compétition avec l'ATP pour la liaison à la tyrosine kinase. Il existe une souche de souris mutantes appelées waved-2 (du fait de l'aspect ondulé de leurs poils) chez lesquelles EGF-R n'est pas supprimé, mais a une faible activité égale à environ $10 \%$ de celle des souris normales. Enfin, il est possible d'utiliser un oligonucléotide antisens pour bloquer l'ARNm de EGF-R.

La première étude démontrant que deux effets essentiels de l'ET - la fibrogenèse et la contractilité vasculaire - dépendent de l'activation de EGF-R est celle de M. Flamant et al. [1]. Pour étudier le premier effet, ces auteurs ont utilisé une souche de souris transgéniques chez lesquelles le gène codant pour la luciférase est sous le contrôle du promoteur de la chaîne $\alpha 2$ du collagène I. L'activité de l'enzyme est un indice de la synthèse du collagène I. L'ET, ajoutée au milieu d'incubation d'anneaux aortiques fraîche- 